inclusions on electron microscopy. Given the combination of interstitial lung disease, skin rash and likely vasculopathy, SAVI was suspected. This was confirmed on genetic testing with a heterozygous somatic mutation (c.463G $>$ A, p. V155M) in exon 5 of the TMEM173, the gene encoding STING.

Treatment Treatment with pulsed methylprednisolone was commenced without improvement. He gained weight with supplemental feeding but had persistent tachynoea, subsequently becoming hypoxaemic requiring low flow oxygen therapy. He commenced on a trial of monthly intravenous immunoglobulin (IVIg) with evidence of clinical efficacy awaited. We are considering the use of the Janus kinase inhibitor, baricitinib, as a specific targeted therapy to block interferon signalling.

Conclusion SAVI is a recently described interferonopathy in which lung involvement is a major clinical feature with consequent significant morbidity and mortality. Twelve patients have been reported so far in the literature, with overall poor response to glucocorticoids and disease modifying anti-rheumatic drugs. In the context of failure to thrive, fevers, rash and interstitial lung disease in early life, we urge clinicians to consider SAVI as a differential diagnosis and to seek testing for TMEM173 mutations.

\section{P97 UPTAKE OF THE EMERGENCY SALBUTAMOL INHALER IN NORTH EAST ENGLAND SECONDARY SCHOOLS FOLLOWING AMENDMENT OF THE HUMAN MEDICINES REGULATIONS}

W Funston, SJ Howard. Health Education North East, Newcastle Upon Tyne, UK

\subsection{6/thoraxjnl-2015-207770.234}

Introduction and objectives As a result of amendments to The Human Medicines Regulations 2012, schools have been permitted since $1^{\text {st }}$ October 2014 to purchase salbutamol inhalers to be used by children diagnosed with asthma and prescribed an inhaler, where parents have given written permission for the emergency inhaler to be used. This regulatory change may represent a useful step in facilitating access to emergency asthma treatment in schools.

This study provides the first published data on the number of schools that have availed of this new power, through an assessment of uptake of the emergency salbutamol inhaler in secondary schools in North East England.

Methods We compiled a list of all free-to attend schools within the 12 local authorities in North East England using listings on local authority websites. We limited our study to schools which served 16 year old mainstream pupils in order to aid interpretation of our results. Postal letters were sent to invite the included schools to complete a brief online or postal questionnaire asking if the school had an emergency salbutamol inhaler for use by pupils in an asthma emergency. Data was collected between November 2014 and May 2015.

Results Of 153 schools included in the study, 103 questionnaire responses were received. We excluded the response of 1 school due to lack of clarity. Of the remaining 102 responses, 45 (44\%) indicated that the school had an emergency salbutamol inhaler available, while 57 (56\%) indicated that the school did not have such an inhaler. The proportion of schools in which emergency salbutamol inhalers were available varied by local authority from $0 \%$ to $71 \%$.

Conclusions Despite the change in legislation, 56\% of schools included in this study did not possess an emergency salbutamol inhaler. More needs to be done to increase the level of uptake of the emergency salbutamol inhaler to enable schools to better respond to asthma emergencies.

\section{P98 THE RELATIONSHIP BETWEEN INVASIVE AND NON- INVASIVE MEASURES OF INFLAMMATION IN CHILDREN WITH SEVERE THERAPY-RESISTANT ASTHMA}

S Mckeon, S Saglani, A Bush, L Fleming. Respiratory Paediatrics, Royal Brompton Hospital London and NHLI Imperial College London, London, UK

\subsection{6/thoraxjnl-2015-207770.235}

Background Children with severe therapy-resistant asthma (STRA) are refractory to treatment despite optimal management. Assessment of airway inflammation to phenotype these patients can enable targeted therapy. Samples obtained at bronchoscopy provide the most direct measure of lower airway inflammation; however, non-invasive measures (induced sputum and exhaled nitric oxide $(\mathrm{FeNO})$ ) are of greater clinical utility. We have previously demonstrated a poor relationship between blood and bronchoalveolar lavage (BAL) eosinophilic phenotype using clinical cut-offs for children (blood eosinophils $1.0 \times 10^{9} / \mathrm{L}$ ). ${ }^{1}$ Recent studies of the anti-IL-5 antibody mepolizumab have used a lower cut point $\left(0.3 \times 10^{9} / \mathrm{L}\right)$ for blood eosinophils. ${ }^{2}$ The aim of this study was to assess the concordance between BAL and non-invasive measures of inflammation.

Methods 113 children (aged 4-17 years) with STRA underwent bronchoscopy at the Royal Brompton Hospital. They had all previously been assessed and potentially modifiable factors such as poor adherence had been addressed. Inflammation was measured invasively using BAL cytology and non-invasively by blood eosinophils, induced sputum cytology, and FeNO. The eosinophilic phenotype was defined as BAL eosinophils $>1.19 \%$; blood eosinophils $\geq 0.3 \times 10^{9} / \mathrm{L}$; sputum eosinophils $\geq 2.5 \%$; and FeNO $>35 \mathrm{ppb}$. The relationship between measures was assessed using Spearman rank correlation and Receiver Operator Characteristic (ROC) curves were constructed to determine which cut points best determined BAL eosinophilia and positive and negative predictive values (PPV and NPV) calculated.

Results The predominant phenotype in all samples was eosinophilic. There was $75.6-77.8 \%$ concordance between the eosinophilic phenotype in BAL and each of the non-invasive measures.

\begin{tabular}{|c|c|c|c|c|}
\hline & \multicolumn{4}{|c|}{ Predicting BAL eosinophilia >1.19\% } \\
\hline & Sensitivity (\%) & Specificity (\%) & PPV (\%) & NPV (\%) \\
\hline \multicolumn{5}{|c|}{ Blood eosinophils $\left(\times 10^{9} / L\right)$} \\
\hline$>0.15$ & 89.8 & 60 & 84.1 & 71.4 \\
\hline$\geq 0.3$ & 80 & 68 & 85.2 & 56.7 \\
\hline$>0.45$ & 59.3 & 84 & 89.7 & 46.7 \\
\hline \multicolumn{5}{|c|}{ Sputum eosinophils,\% } \\
\hline$>0$ & 90.9 & 38.5 & 78.9 & 62.5 \\
\hline$\geq 2.5$ & 78.8 & 61.5 & 83.9 & 53.3 \\
\hline$>5$ & 63.6 & 69.2 & 84 & 42.9 \\
\hline \multicolumn{5}{|c|}{ FeNO (ppb) } \\
\hline$>23$ & 84.9 & 62.5 & 83.3 & 65.2 \\
\hline$>35$ & 79.2 & 75 & 87.5 & 62.1 \\
\hline
\end{tabular}

PPV = positive predictive value, $\mathrm{NPV}=$ negative predictive value 
Blood and BAL eosinophils had the strongest correlation $(\mathrm{r}=$ $0.57, \mathrm{p}<0.001, \mathrm{n}=84)$. Weaker correlations were found between the other measures. The most promising predictor of BAL eosinophilia was a blood eosinophil count of $0.15 \times 10^{9} / \mathrm{L}$ (PPV 84.1, NPV 71.4) (Table 1).

Conclusions These results suggest that blood eosinophils at a lower cut-point may be a useful measure of lower airway inflammation. However, this is still a relatively invasive test in children and there is little data available about longitudinal stability of blood eosinophils.

\section{REFERENCES}

1 Ullmann N, et al. Allergy 2013;68:402

2 Pavord ID, et al. Lancet 2012;380:651

\section{P99 COLONISATION WITH FILAMENTOUS FUNGI AND ACUTE EXACERBATIONS IN CHILDREN WITH ASTHMA}

KG Staley, CH Pashley, J Satchwell, AJ Wardlaw, EA Gaillard. University of Leicester, Leicester, UK

\subsection{6/thoraxjnl-2015-207770.236}

Background Children with asthma are frequently sensitised to fungi and recent observations suggest that fungal sensitisation may be associated with more severe asthma in children. ${ }^{1,2}$ Aspergillus fumigatus airway colonisation in adults with asthma is associated with reduced lung function. ${ }^{3}$ There is a paucity of data onfungal colonisation in children with asthma. The role of fungi in exacerbation prone asthma has not been previously investigated. Our study aim was to evaluate the association between fungal airway colonisation and exacerbation prone asthma in children.

Methods Children aged 5-16 years with stable asthma who attended for a routine hospital outpatient appointment and children with an acute exacerbation of asthma who attended for urgent care to an acute admissions unit were recruited to the study. We obtained a sputum sample either via nebulisation with hypertonic saline in children with stable asthma or nebulisation with $0.9 \%$ saline in children with acute asthma. Sputum culture was focused to detect filamentous fungi, in particular Apergillus and Penicillium species. ${ }^{3,4}$ Culture and sensitisation results were compared with clinical assessment data.

\begin{tabular}{llll}
$\begin{array}{l}\text { Abstract P99 Table } 1 \\
\text { and sasthma }\end{array}$ & Demographics and fungi isolated in acute \\
\hline & $\begin{array}{l}\text { Acute asthma } \\
\mathbf{n}=26\end{array}$ & $\begin{array}{l}\text { Stable asthma } \\
\mathbf{n}=29\end{array}$ & P value* \\
\hline Median age in years (range) & $8(5-15)$ & $10.5(5-16)$ & 0.122 \\
Male (\%) & $17(65.4)$ & $20(68.9)$ & 0.778 \\
Filamentous fungi culture positive $\mathbf{n}(\%)$ & $11(42.3)$ & $5(17.3)$ & 0.041 \\
A. fumigatus & 9 & 4 & \\
A niger & 0 & 1 & \\
Penicillium & 2 & 1 & \\
\hline${ }^{*}$ Mann Whitney or Chi squared test. & & & \\
\hline
\end{tabular}

Results Fifty five children were recruited to the study; 26 with acute asthma and 29 with stable asthma (17 BTS step 4-5). There was no difference in demographics between the two groups (Table 1). Sixteen children (29\%) were culture positive for filamentous fungi, either Aspergillus fumigatus (81.3\%) or Penicillium (18.7\%). One child with stable asthma harboured two different filamentous fungi, $A$. niger and $A$ fumigatus. Children with acute asthma were more likely to be culture positive for filamentous fungi than children with stable asthma $(42.3 \%$, n $=11 \mathrm{v} 17.2 \%, \mathrm{n}=5$ respectively, $\mathrm{p}=0.041)$. Of the five children with stable asthma who were culture positive for filamentous fungi, three were BTS step 4-5.

Conclusions Significantly more children with acute asthma had filamentous fungi isolated from their sputum compared to children with stable asthma. Aspergillus fumigatus was the most common fungus isolated. The potential role of fungal airway colonisation in triggering asthma attacks in children merits further investigation.

\section{REFERENCES}

1 Castanhinha $S$, Sherburn $R$, Walker $S$, et al. Pediatric severe asthma with fungal sensitization is mediated by steroid-resistant IL-33. J Allergy Clin Immunol 2015:136:312-322

2 Vicencio AG, Santiago MT, Tsirilakis $K$, et al. Fungal sensitization in childhood persistent asthma is associated with disease severity. Pediatr Pulmonol 2014;49:8-14

3 Fairs A, Agbetile J, Hargadon B, et al. IgE sensitization to Aspergillus fumigatus is associated with reduced lung function in asthma. Am J Respir Crit Care Med 2010;182:1362-8

4 Agbetile J, Fairs A, Desai D, et al. Isolation of filamentous fungi from sputum in asthma is associated with reduced post-bronchodilator FEV1. Clin Exp Allergy 2012;42:782-91

\section{P100 IMPROVING PAEDIATRICS' PRESSURISED METERED DOSE INHALER TECHNIQUE AND ASTHMA CONTROL: INHALER VERBAL COUNSELLING VS. TRAINHALER}

${ }^{1}$ WG Ammari, ${ }^{1} \mathrm{NK}$ Al-Hyari, ${ }^{2} \mathrm{~N}$ Obeidat, ${ }^{2} \mathrm{M}$ Khater, ${ }^{3} \mathrm{~A}$ Sabouba, ${ }^{4} \mathrm{M}$ Sanders. ${ }^{1}$ Faculty of Pharmacy and Medical Sciences, Al-Ahliyya Amman University, Amman, Jordan; ${ }^{2} J o r d a n$ University Hospital, Amman, Jordan; ${ }^{3}$ Al-Hussain Public Hospital, Amman, Jordan; ${ }^{4}$ Clement Clarke International, Harlow, UK

\subsection{6/thoraxjnl-2015-207770.237}

Introduction Verbal counselling (VC) is commonly used to train patients on correct inhaler technique. Patients forget the good inhaler use with time. Trainhaler $(\mathrm{TH})$, Clement Clarke, UK, is a novel pressurised metered dose inhaler (MDI) training tool designed with feedback mechanisms to train patients coordinate releasing the aerosol with using a slow and deep inhalation flow (IF) through their MDI. Our aim was to compare VC with TH in children with asthma.

Methods Ethical approval was obtained and all children and their parents gave signed consent. At visit 1 , asthmatic children, age 7-17 years, with an MDI hand-lung coordination problem including an IF > $60 \mathrm{l} / \mathrm{min}$ were randomised into either the VC group that received verbal MDI training with emphasis on using a slow and deep IF; or into the TH group that were trained onand given $\mathrm{TH}$ to practice at home. Children with correct MDI technique and IF $\leq 60 \mathrm{l} / \mathrm{min}$ formed the control group (CT). An 11-step MDI technique, peak IF through the inhaler and Asthma Control Questionnaire (ACQ) were evaluated. All subjects returned after 6 to 8 weeks (visit 2) for re-evaluation.

Results Thirty children took part. Table 1 presents the study outcomes. All VC and TH had correct MDI steps and slow IF posttraining at visit 1 . Unlike CT, Wilcoxon test showed a significant decrease $(p<0.01)$ in the incorrect MDI steps between visits 1 and 2, within VC and TH. Mann-Whitney test showed a significant difference $(\mathrm{p}<0.01)$ in the incorrect MDI steps between the CT and both intervention groups at visit 1 , but no significant difference $(p>0.05)$ was found at visit 2 . Paired t-test showed 